\title{
Anti-neoplastic effects of non-digestible carbohydrates on WNT signalling in the bowel: a randomised controlled dietary intervention
}

\author{
F. Malcomson ${ }^{1}$, N. Willis ${ }^{1}$, I. McCallum ${ }^{1}$, L. Xie $^{1}$, W. Leung ${ }^{2}$, S. Kelly ${ }^{1}$, M. Bradburn ${ }^{1}$, \\ N. J. Belshaw ${ }^{2}$, I. T. Johnson ${ }^{2}$ and J. C. Mathers ${ }^{1}$ \\ ${ }^{1}$ Human Nutrition Research Centre, Institute for Ageing and Health, Newcastle University, Newcastle upon Tyne NE4 5PL, \\ UK, ${ }^{2}$ Institute of Food Research, Norwich Research Park, Norfolk NR4 7UA, UK
}

Colorectal cancer (CRC) is the third most common cancer in the UK. The majority of CRC cases develop sporadically ${ }^{(1)}$, and the WNT signalling pathway is implicated in the aetiology of both sporadic and inherited forms ${ }^{(2)}$. Environmental factors, including diet, modify CRC risk and non-digestible carbohydrates (NDCs), such as dietary fibre and resistant starch (RS), appear to be protective ${ }^{(3)}$. These beneficial effects are believed to result from the production of butyrate, a short-chain fatty acid, by colonic bacteria and evidence exists for the modulation of WNT signalling by butyrate. This study aimed to investigate the effects of supplementing healthy participants with NDCs on WNT-related gene expression and its functional outcomes in the bowel. It is hypothesised that increasing NDC intake will increase colonic butyrate concentrations and modulate WNT signalling.

75 healthy participants were recruited as part of the DISC Study and supplemented with RS and/or polydextrose or placebo for 7 weeks in a $2 * 2$ factorial design. Rectal mucosal biopsies were collected pre- and post-intervention. RNA was extracted from OCT-embedded biopsies and reverse transcribed to cDNA for the quantification of CCND1, MYC and SFRP1 expression in duplicate by quantitative PCR.
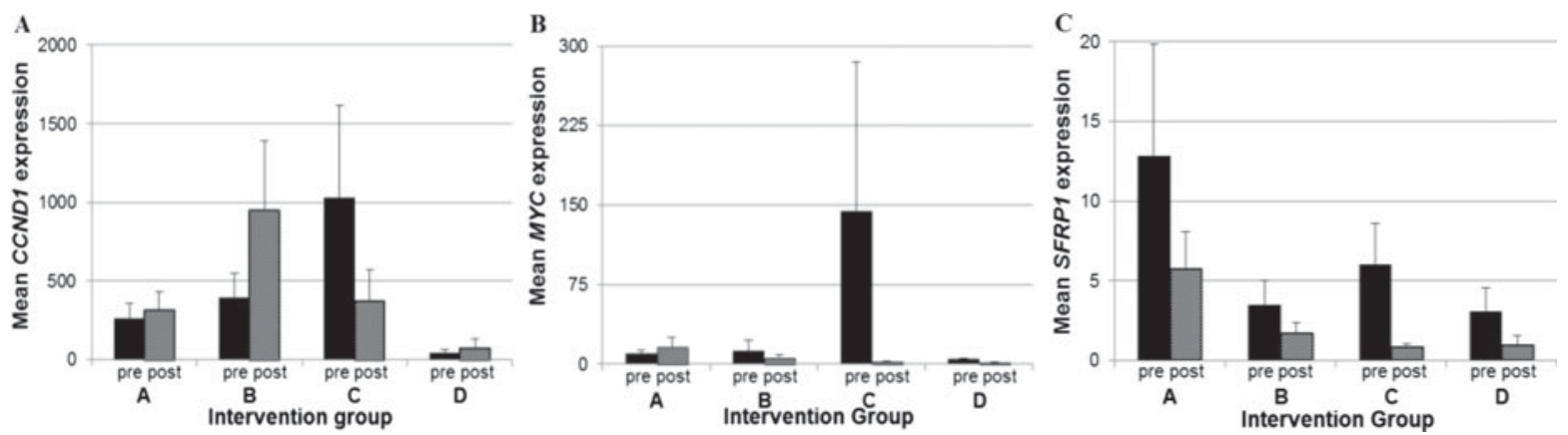

Fig. 1. Mean expression of $C C N D I$ (A), MYC (B) and SFRPI (C), expressed as adjusted copies $\left(2^{-\Delta C t} \times 1000\right)$ relative to the $18 S$ housekeeping gene $(n=36)$

Preliminary results $(n=36)$ indicate differences in the expression of CCND1, MYC and SFRP1 post-intervention for all four groups (Fig. 1). No statistically significant differences in baseline (pre-intervention) expression of $C C N D 1(P=0.189), M Y C(P=0.447)$ or SFRP1 $(P=0.338)$ between the intervention groups were observed following analysis using the ANOVA General Linear Model. This study will be un-blinded in May to determine if any effects of NDC supplementation on WNT-related gene expression exist. In addition, effects of the intervention on proliferation and apoptosis, two functional outcomes of WNT signalling that are deregulated in CRC, are currently being investigated.

This study was funded by the BBSRC (BB/H005013/1). Ethical approval for this project, as part of a larger application, was granted on $10^{\text {th }}$ December 2009 (REC No. 09/H0907/77).

1. Fearnhead NS, Wilding JL and Bodmer WF (2002) Br Med Bull 64, 27-43.

2. Bienz $\mathrm{M}$ and Clevers H (2000) Cell 103, 311-20.

3. Lipkin M, Reddy B, Newmark H et al. (1999) Annu Rev Nutr 19, 545-586.

4. Bordonaro M, Lazarova DL \& Sartorelli AC (2008) Cell Cycle 9, 1178-83. 